common clinical presentations.Hypergammaglobulinemia is diagnostic of increased CD4-CD8-T cells in peripheral blood. Often it is susceptible to B Cell Lymphoma. In our presentation, we wanted to draw attention to this issue by presenting two different cases, one in the differential diagnosis of lymphoma and the other in the diagnosis of ALPS after long-term lymphoma treatment.

A 6-year-old girl presented in April 2012 with complaints of swelling and night sweats on the left side of the neck. On physical examination, hepatosplenomegaly was absent, and multiple lenadenopathies were detected in the left cervical chain. No agent was detected in terms of infectious pathologies. There were no pathological cells in bone marrow aspiration performed for malignant diseases, but multiple lymph nodes with cervical, supraclavicular and intra-abdominal hypermetabolic activity were detected in PET imaging. She was diagnosed as Mixed type Hodgkin's Lymphoma by supraclavicular lymph node excision. After ABVD and COPP treatment, radiotherapy was applied to the abdomen, neck and mediastinum. While regression was seen in PET imaging after treatment, the disease was progressed by bone marrow activation and lung parenchymal involvement.Autologous Bone Marrow Transplantation was performed in August 2014, but recurrence was detected for the second time in January 2015. In the follow-up, although the treatment of Brentuximab, Gemcitabine, Paclitaxel and Nivolumab were applied, the disease progressed. Double T Negative Cells 5.3\% were detected in immunophenotyping, which was sent for possible immune deficiencies. The history of lymphadenopathy, the predisposition of B lymphoma, the rate of DNT above $2.5 \%$, the presence of a consanguineous marriage between parents and the rate of DNT $11.6 \%$ of the sisters who had no complaints, suggested the possibility of ALPS (Autoimmune Lymphoproliferative Syndrome) in the patient. The patient, whose m-Tor inhibitor Sirolimus treatment was started, has been on follow-up for 3 years. Genetic tests of the patient were sent.

A three-year-old male patient was diagnosed as Acute ITP by detecting thrombocytopenia in his examinations with the appearance of bruises on his body after infection. No pathology was observed in bone marrow aspiration.

The response to IVIG and pulse steroid treatments was not good. Due to splenomegaly, coombs positive hemolytic anemia and thrombocytopenia continuing in the follow-up of the patient, DNT cell count was found 7.9\% considering ALPS. Genetic tests of the patient were sent. Sirolimus treatment was started for the patient who did not respond well to MMF and oral steroid.

\section{OVERVIEW ON THE CURRENT MANAGEMENT GUIDELINES OF IMMUNE THROMBOCYTOPENIA IN CHILDREN}

${ }^{1}$ Vasile-Eduard Roşu*, ${ }^{1,2}$ Solange-Tamara Roşu, ${ }^{1,2}$ Anca-Viorica Ivanov, ${ }^{1,2}$ Mocanu AdrianaMaria, 1,2Spoială Lia-Elena, 1,2Duşa Cristian-Petru, 1,2Ingrith Miron, 1,2Cristina Gavrilovici. 'Grigore T. Popa' Medicine and Pharmacy University lasi, Romania; 'Sfânta Maria' Emergency Clinical Hospital for Children lasi, Romania

\subsection{6/archdischild-2021-europaediatrics.298}

Our aim is to identify, analyze and compare the international guidelines or national consensus reports on the management of immune thrombocytopenia in children.(ITP).
We performed a systematic search on PubMed database using keywords:'imune thrombocytopenia in children AND ['children' OR 'pediatric' OR 'paediatric'] AND ['guideline' OR 'consensus'] between 1992 (first guideline) and 2020. We excluded publications written in other languages than English or French and animal studies. A total of 54 papers have been initially found. After exclusion of those that were not relevant or other types of publications than guidelines or consensuses (reviews, case series, case reports) we ended up in gathering 44 publications. After full text screening, we excluded papers that did not particularly refer to ITP guidelines but to quality of life, adherence to treatment etc. Finally 6 papers have been found to meet such strict criteria.

They are only six countries in the world published having a specific ITP published in Pub Med:USA with American Society of Oncology Guidelines for Immune Thrombocytopenia, Great Britain, Spain, Italy, Argentine and Japan.

The USA and Italian Guidelines recommend for children newly diagnosed with ITP without bleeding or minor bleeding observation rather than corticosteroids and Immunoglobuline IV (USA). For Children with non-life threating mucosal bleeding the american guideline suggest cortycotherapy no longer than 7 days. For the forms non-responsive at the first line the treatament, the American and Spanish Guidelines indicate thrombepoetin receptors agonist( TPO-RA-Eltrompobag) rather than Rituximab and Splenectomy. According to the spanish guideline cortycotherapy is the first choice therapeutic. Generally the primary goals of these guidelines are to review and implement evident based-recommandations. Other treatments include Azathiopirine, Cyclophosphamide, Cyclosporine A, Dapsone, Danazol and Myofenolate of Mofetil. In 2018 a joint working group (JWG) of several hematology societies (Germany, Switzerland, Austria) published a European Guideline for adults with ITP but more limitate for children with ITP ( no standard trateament for chronic ITP at children).

The splenectomy is universally the last options for treatament in ITP at children.

The general purpose of the Guidelines are the implement new therapies (Eltrompobag/Romiplostim) at children because they are rather than cortycotherapy and immunoglobuline iv (which are important side-effects and expansive bugets ). At children for Eltrompobag are raported minor or moderate side-effects and no for long therm.

\section{CHILDHOOD NEUTROPENIA - OUR EXPERIENCE}

Nuša Matijašič ${ }^{*}$ Domagoj Buljan, Izabela Kranjčec, Gordana Jakovljević. Children's Hospital Zagreb

10.1136/archdischild-2021-europaediatrics.299

Our goal was to describe demographic characteristics, course and outcome of neutropenic patients.

We used descriptive statistics to demonstrate demographic and clinical characteristics of patients who were referred to pediatric hematologist's examination or hospitalized in the Department of Hematology and Oncology, Children's Hospital Zagreb, due to neutropenia from January 1st, 2009 to December 31st, 2019. Oncology patients with febrile neutropenia were excluded from the study.

Altogether, 100 children were examined or hospitalized in our Department during the 11-year period due to neutropenia, of which 56 were males, with an average age of 3.48 
years. The cause of a low absolute neutrophil count (ANC) was autoimmune neutropenia in 37 patients, 31 had chronic idiopathic neutropenia, 27 had infectious neutropenia, 3 had cyclic neutropenia, while 2 were diagnosed with alloimmune neonatal neutropenia. The mean value of ANC at presentation was $732 / \mathrm{mm} 3$, and the mean lowest ANC detected during disease course was $600 / \mathrm{mm} 3$. Among the 5 diagnostic subgroups, both values were the lowest in patients with cyclic neutropenia in which mean ANC at presentation was $420 / \mathrm{mm} 3$ and mean lowest ANC detected during disease course was 180/ $\mathrm{mm} 3$.

Granulocyte colony-stimulating factor (GCS-F) was given to only 3 patients, whose mean ANC at presentation was 140/ $\mathrm{mm} 3$. Besides the laboratory finding of persistent severe neutropenia, all 3 patients were prone to recurrent infections. Twenty-three patients had additional cytopenia, out of which 4 had pancytopenia, 13 had anemia and 6 patients had thrombocytopenia. Among patients with additional cytopenia, 5 had positive anti-granulocyte antibodies (21.7\%). The mean time to disease resolution was 10.2 months, being the longest in the cyclic neutropenia subgroup, while patients with infectious neutropenia mainly recovered after 21.3 days. The mean follow-up time was 1.6 years.

According to data from our Department, neutropenia is most commonly diagnosed in pre-school children, boys being more frequently affected than girls. More than one third of patients have positive anti-granulocyte antibodies. However, the condition is usually benign, it resolves mainly spontaneously in less than a year, and patients generally do not require G-CSF.

\section{IMMUNOTHERAPY IN HIGH RISK NEUROBLASTOMA - TREATMENT ALGORITHM OF COMPLICATIONS}

Jasminka Stepan-Giljevic*, Gordana Jakovljevic, Aleksandara Bonevski, Filip Jadrijevic Cvrlje, Nada Rajacic, Maja Pavlovic, Izabela Kranjcec. Department of hematology and oncology „Dr Mladen Cepulic', Children's Hospital Zagreb

\subsection{6/archdischild-2021-europaediatrics.300}

We report our center experience in immunotherapy of high risk neuroblastoma, in particular concerning the most common side effects and their management.

Multidisciplinary team approach will be discussed as well, as it is especially important in care for these patients.

Retrospective review of hospital medical data basis High risk neuroblastoma patients receive intensive multimodal therapy that includes induction, consolidation, and postconsolidation phases. The postconsolidation or maintenance phase implies immunotherapy with anti-GD2 monoclonal antibody dinutuximab beta, applied with the purpose of eliminating any residual tumor cells that may exist after induction and consolidation treatment (multiagent chemotherapy, surgery, high-dose chemotherapy and autologous stem cell transplant).

During the 3 -year period $(2017$ - 2019) 8 high risk neuroblastoma patients received immunotherapy with dinutixumab beta in our center. Patients were in remission confirmed by different methods of investigation. Dinutuximab beta was applied in hospital conditions, as a 10-day continuous infusion, with premedication and concomitant medications that included crystalloid fluids, various pain medications, antihistamines, antiemetics and antipyretics, requiring double lumen central venous catheter and continuous monitoring of vital functions.

Most of our patients achieved adequate pain control with gabapentin and titrated doses of opioids. Fever was a common side effect easily managed by NSAIDs. In case of allergic reaction the rate of dinutuximab beta was decreased by half and restored to its full rate after resolution of symptoms. In the meantime additional doses of antihistamines, oxygen, salbutamol and racemic epinephrine were applied depending on the clinical condition.

Special care was dedicated to fluid balance supervision due to the risk of capillary leak syndrome. Although 5 of our patients had some degree of capillary leak, only one patient developed significant hypotension which was recognized and treated in timely manner with fluid boluses and epinephrine titrated to effect. Upon resolution of hypotension, the drug infusion was restarted at 50\% rate during 2 hours and after that in the absence of recurrent hypotension increased to the full rate.

Postconsolidation immunotherapy with dinutuximab beta has become the standard of care for high-risk neuroblastoma patients. The benefits of this kind of therapy do not come without risks. Having front-line providers who are familiar with immunotherapy and its associated toxicities is critical to the safe and consistent administration of this complex therapy. The team includes physicians of different specialities, nurse practitioners, physician assistants and bedside nurses.

\section{DIAGNOSTICS AND TREATMENT OF CHILDHOOD MALIGNANT LIVER TUMORS - A SINGLE CENTER EXPERIENCE}

Jasminka Stepan-Giljevic*, Domagoj Buljan, Aleksandara Bonevski, Nada Rajacic, Gordana Jakovljevic, Filip Jadrijevic Cvrlje, Maja Pavlovic, Izabela Kranjcec. Department of hematology and oncology „Dr Mladen Cepulic', Children's Hospital Zagreb

\subsection{6/archdischild-2021-europaediatrics.301}

We report our center experience in medical management of malignant childhood liver tumors Retrospective review of hospital medical data basis In the period of 19 years 13 patients were diagnosed and treated in our institution due to malignant liver tumor (8 males and 5 females). Average age at diagnosis was 4,04 years. 3 of them were born prematurely.

Histopathological type of the tumor corresponded to hepatoblastoma in all cases, except one which is the case of hepatocellular carcinoma. The most frequent PRETEXT group was stage 2. Radiological imagaing and biopsy were important in making the correct diagnosis. The usual medical management consisted of neoadjuvant chemotherapy and surgical procedure followed by adjuvant chemotherapy. Overall survival is $76,9 \%$ and the average follow-up period 71,1 months.

Malignant childhood liver tumors are challenging to manage necessitating multidisciplinary approach and collaboration.

\section{4-MONTH-OLD BOY WITH REFRACTORY THROMBOCYTOPENIA AND WISKOTT-ALDRICH SYNDROME - CASE REPORT}

Agnieszka Słowińska*, Karolina Bohdankiewicz, Paulina Dziuba. Wojewódzki Specjalistyczny Szpital Dziecięcy w Olsztynie

10.1136/archdischild-2021-europaediatrics.302 